\title{
Stiff-Person Syndrome: A Case Report
}

\author{
Prabhjot Kaur Manes ${ }^{\mathrm{a}, \mathrm{b}}$, Saif Mohammad Aslama , Melvin Varghese ${ }^{\mathrm{a}}$, \\ Lia Elipulikattu ${ }^{\mathrm{a}}$
}

\begin{abstract}
This is a case report of stiff-person syndrome (SPS) with episodes of paroxysmal autonomic dysfunction. A 67-year-old female with hypothyroidism, anxiety and hypertension presented with shortness of breath and diaphoresis for 1 day. The patient had been bedbound for several years due to muscle rigidity. She had two episodes of paroxysmal autonomic dysfunction treated with Ativan which resolved. Her anti-glutamic acid decarboxylase (GAD) antibody became abnormally high at $>250$. She was treated with IVIG $30 \mathrm{~g}$ daily for 5 days as an inpatient and was discharged on 3 monthly IVIG treatment $1 \mathrm{~g} / \mathrm{kg}$ $\times 2$ days. This case report describes SPS with paroxysmal autonomic dysfunction.
\end{abstract}

Keywords: Stiff-person syndrome; Paroxysmal autonomic dysfunction

\section{Introduction}

Stiff-person syndrome (SPS) (formally known as stiff-man syndrome) was first diagnosed by Moersch and Waltman in 1956 after reviewing 14 patients over 27 years. It is an extremely rare neuro-immunological disorder which is characterized by progressive axial muscle rigidity and stiffness accompanied by painful episodes of muscle spasms [1]. Anti-glutamic acid decarboxylase (GAD65) antibody is very specific for SPS and is found to be positive in the blood and CSF of $60 \%$ of SPS patients $[2,3]$. In some cases, SPS is a paraneoplastic syndrome associated with neoplasm such as breast cancer [3]. It is also often associated with type 1 diabetes mellitus (T1DM).

\section{Case Report}

A 67-year-old bedbound African American female with history

Manuscript accepted for publication August 11, 2016

${ }^{a}$ The Brooklyn Hospital Center, Brooklyn, NY, USA

${ }^{b}$ Corresponding Author: Prabhjot Kaur Manes, The Brooklyn Hospital Center, 121 Dekalb Ave., Brooklyn, NY 11201, USA.

Email: manesprabhjot@yahoo.com

doi: http://dx.doi.org/10.14740/jmc2619w of hypothyroidism, anxiety and hypertension presented to the emergency department with shortness of breath, diaphoresis for 1 day and one episode of diarrhea several days prior. She complained of left arm pain, stating she was unable to move it and did not allow further examination. The patient had a surgical history of left breast cyst removal and left wrist cyst removal. Report from the patient's daughter stated that she had been bedbound for several years. The patient was on metoprolol, synthroid, buspirone, baclofen and motrin. Her allergies include aspirin and shellfish. Her family history is significant for tuberculosis, diabetes mellitus in her father and asthma in her daughter. She denied history of smoking, alcohol or illicit drug use.

On examination, patient is an elderly, frail, bedbound woman oriented to time, place and person with multiple visible macules over her arms. Cardiovascular and pulmonary examinations were significant for tachycardia and tachypnea, but were otherwise unremarkable. Abdomen was non-tender and rigid throughout. Musculoskeletal exam showed bilateral lower extremity rigidity, with no edema. Patient declined upper extremity examination due to pain. Bilateral gluteal abrasions with areas of hypopigmentation were also noted.

Laboratory analysis showed WBC of $17,400 / \mathrm{cm}^{3}$ with left shift and hemoglobin and hematocrit were within normal limits. Her BUN was within normal limits, but her creatinine was elevated to $1.2 \mathrm{mg} / \mathrm{dL}$ (baseline $0.7 \mathrm{mg} / \mathrm{dL}$ ). Lactic acid was elevated at $12.4 \mathrm{mmol} / \mathrm{L}$. Bicarbonate was low at $11 \mathrm{mmol} /$ $\mathrm{dL}$, and phosphate was high at $5.4 \mathrm{mg} / \mathrm{dL}$. Arterial blood gas showed $\mathrm{pH} 7.43, \mathrm{pCO}_{2} 34 \mathrm{~mm} \mathrm{Hg}, \mathrm{pO}_{2} 54 \mathrm{~mm} \mathrm{Hg}, \mathrm{HCO}_{3}$ $23 \mathrm{mmol} / \mathrm{L}$, and $\mathrm{O}_{2}$ saturation $89 \%$. Urine collection was not possible due to the extensive contraction of the patient's legs. Chest X-ray showed no evidence of infiltration or suspicious mass in lungs. Patient was given one dose of zosyn, vancomycin, and cefepime for possible sepsis. Blood cultures displayed Gram positive cocci in clusters and PNA fish grew coagulate negative Staphylococcus, most likely a contaminant. Infectious disease was consulted and recommended urine culture, urinalysis, CPK, TSH levels and to start empiric treatment with ceftriaxone. Patient refused imaging of abdomen pelvis and further blood draws.

On day 3 of admission, she had her first paroxysmal autonomic dysfunction during an attempt at straight catheterization for a urine sample, and patient became agitated screaming, "This is how I died before" and "Don't touch me, don't move my legs." Patient became tachycardic, tachypnic, diaphoretic and hyperpyretic. She was noted to have significant hypertonia of lower extremities and eyes rolling back into her head. 
In addition, patient began to heave, contracting her abdomen, as though in Valsalva. Throughout the episode the patient remained alert, oriented and verbal. ICU team was promptly called to evaluate patient for intubation and upgrade to a higher level of care.

In ICU, patient remained agitated, tachycardic and tachypnic. She was given a trial of benzodiazepine $2 \mathrm{mg}$ Ativan IV push, to which she responded very well. She immediately became less agitated, her respiratory rate improved, oxygen saturation remained at $97 \%$, but she was still tachypnic. After this episode, the suspicion of SPS was high and CT of head/ neck was ordered, as well as anti-GAD antibody titers. AntiGAD antibody was reported positive (> $250 \mathrm{IU} / \mathrm{mL})$. Lumbar puncture confirmed presence of anti-GAD antibody, with all other associated autoimmune antibodies, including TPO, and rheumatology panels were noted to be unremarkable. However, patient refused MRI of the brain and C-spine to rule out other neurological diseases such as multiple sclerosis or cervical myelopathy.

On day 9 of admission, patient had another episode of autonomic dysfunction. She was diaphoretic, tachycardic, hypertensive and anxious. She was given valium $5 \mathrm{mg}$ IM and she also received Ativan $4 \mathrm{mg}$ PO. Patient gradually became stable. Her baclofen dose was increased to $20 \mathrm{mg}$ every $6 \mathrm{~h}$, and valium to $5 \mathrm{mg}$ every $6 \mathrm{~h}$. Under neurology recommendation, IVIG $30 \mathrm{~g}$ daily for 5 days was started. At the end of the medication course, patient showed remarkable improvement, with reduced muscle rigidity and increased range of motion. After discharge, she will receive 3 monthly IVIG treatment 1 $\mathrm{g} / \mathrm{kg}$ with the duration of 2 days.

\section{Discussion}

SPS is an extremely rare neuro-immunological disorder. The exact prevalence and incidence of the disease is unknown; however, the prevalence is estimated to be $1 / 1,000,000$ in general population. SPS affects females more than the males, with age of onset in third to sixth decades of life. Although the etiology of the SPS is unknown, it is suspected to have an autoimmune pathogenesis due to the presence of autoantibody against GAD, the rate-limiting enzyme for the synthesis of the gamma-aminobutyric acid (GABA). A possible association has also been suggested with other autoimmune conditions such as T1DM, due to the presence of high levels of anti-GAD antibodies in such patients [4].

The pathophysiology of SPS is also unclear, but has been suggested to be related to the GABAergic pathway. GABA is an inhibitory neurotransmitter in the brain and the spinal cord. The spinal interneuron utilizes GABA to coordinate motor neurons and motor function. Impaired synthesis of GABA neurotransmitter results in continuous firing of spinal motor neurons leading to rigidity, stiffness and spasms which are the hallmark signs of SPS.

SPS manifests as suddenly rigidity and stiffness of the axial muscles in the early stages, with progressive rigidity and stiffness of the proximal muscles in the limbs. These signs and symptoms can be accompanied by episodic painful muscle spasms. The stiffness and the spasms can precipitate falls and fractures. Rarely, patients have been reported to have episodes of paroxysmal autonomic dysfunction causing diaphoresis, tachycardia, tachypnea, hypertension and hyperpyrexia, which result in sudden death [5].

The Dalakas criteria for SPS are used worldwide for the diagnosis of SPS, as follows [6, 7]: 1) stiffness in the axial muscles, prominently in the abdominal and thoracolumbar paraspinal muscle leading to a fixed deformity (hyperlordosis); 2) superimposed painful spasms precipitated by unexpected noises, emotional stress, and tactile stimuli; 3) confirmation of the continuous motor unit activity in agonist and antagonist muscles by electromyography; 4) absence of neurological or cognitive impairments that could explain the stiffness; and 5) positive serology for GAD65 (or amphiphysin) autoantibodies, assessed by immunocytochemistry, western blot or radioimmunoassay.

Investigations for SPS include basic laboratory studies including $\mathrm{CBC}$, testing for anti-GAD antibody in blood and CSF, electromyography, CT scan of the brain and spinal cord to rule out degenerative diseases.

Benzodiazepines are considered an effective initial treatment for SPS, with baclofen used in the patients who are unresponsive to benzodiazepines. Corticosteroid therapy can be used as well. High-dose IVIG therapy has been shown to be well tolerated and effective for the management of SPS [6].

\section{Conclusion}

In this report, we described a patient with SPS with no associated T1DM, who had two episodes of paroxysmal autonomic dysfunction. Her episodes of paroxysmal autonomic dysfunction were treated with Ativan causing significant improvement. After positive anti-GAD antibody screen, patient was treated with IVIG with significant improvement and resolution of symptoms.

\section{References}

1. Moersch FP, Woltman HW. Progressive fluctuating muscular rigidity and spasm ("stiff-man" syndrome); report of a case and some observations in 13 other cases. Proc Staff Meet Mayo Clin. 1956;31(15):421-427.

2. Raju R, Foote J, Banga JP, Hall TR, Padoa CJ, Dalakas MC, Ortqvist E, et al. Analysis of GAD65 autoantibodies in Stiff-Person syndrome patients. J Immunol. 2005;175(11):7755-7762.

3. Duddy ME, Baker MR. Stiff person syndrome. Front Neurol Neurosci. 2009;26:147-165.

4. Levy LM, Dalakas MC, Floeter MK. The stiff-person syndrome: an autoimmune disorder affecting neurotransmission of gamma-aminobutyric acid. Ann Intern Med. 1999;131(7):522-530.

5. Mitsumoto H, Schwartzman MJ, Estes ML, Chou SM, La Franchise EF, De Camilli P, Solimena M. Sudden death and paroxysmal autonomic dysfunction in stiff-man syn- 
drome. J Neurol. 1991;238(2):91-96.

6. Dalakas MC, Fujii M, Li M, Lutfi B, Kyhos J, McElroy B. High-dose intravenous immune globulin for stiff-person syndrome. N Engl J Med. 2001;345(26):1870-1876.
7. Dalakas MC, Fujii M, Li M, McElroy B. The clinical spectrum of anti-GAD antibody-positive patients with stiff-person syndrome. Neurology. 2000;55(10):15311535. 